\title{
Atividade física para gestantes e mulheres no pós- parto: Guia de Atividade Física para a População Brasileira
}

\author{
Physical activity for pregnant and postpartum women: Physical Activity Guidelines \\ for the Brazilian Population
}

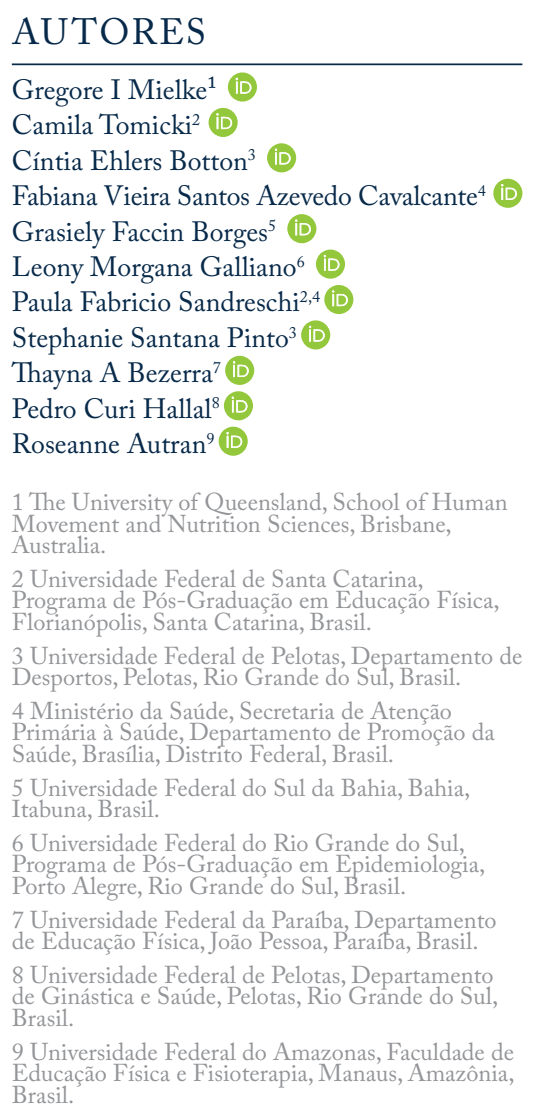

\section{CONTATO}

Gregore I Mielke

g.ivenmielke@uq.edu.au

School of Human Movement and Nutrition Sciences, The University of Queensland | St Lucia Campus Brisbane, QLD 4072, Australia.

DOI

$10.12820 /$ rbafs.26e0217

\section{(cc) BY}

Este trabalho está licenciado com uma Licença Creative Commons - Atribuição 4.0 Internacional.

\section{RESUMO}

Este artigo tem por objetivo apresentar as recomendações de atividade física para gestantes e mulheres no pós-parto desenvolvidas para o Guia de Atividade Física para a População Brasileira. Para isso, utilizou-se uma abordagem de métodos mistos que incluiu quatro etapas: 1) levantamento das diretrizes internacionais mais recentes; 2) revisão narrativa de literatura sobre efeitos da prática de atividade física durante a gestação para a saúde da mulher e do bebê; 3) escuta com gestantes, mulheres no pós-parto, profissionais e pesquisadores; 4) consulta pública. As evidências sumarizadas suportam que a prática de atividade física durante a gestação e no período pós-parto é segura, traz benefícios à saúde da mãe e do bebê, e reduz os riscos de algumas complicações relacionadas à gestação. Gestantes devem ser incentivadas a realizar pelo menos 150 minutos por semana de atividade física de intensidade moderada. Gestantes com contraindicações devem procurar auxílio de profissionais qualificados. Em alguns casos, a prática de atividade física durante a gestação não é recomendada. Este documento servirá como ferramenta para nortear profissionais de saúde que atuam com gestantes e mulheres no pós-parto e irá orientar a população-alvo quanto a prática de atividade física.

Palavras-chave: Atividade física; Gestação; População; Exercício físico.

\section{ABSTRACT}

This manuscript aims to present the recommendations of physical activity for pregnant and postpartum women developed for the Physical Activity Guidelines for the Brazilian Population. A mixed-method approach that included four steps was used: 1) review of the most recent international guidelines for physical activity during pregnancy; 2) narrative literature review on the effects of physical activity during pregnancy on the health of the woman and the baby; 3) consultation with pregnant women, postpartum women, professionals and researchers; 4) public consultation. The summarized evidence supports that physical activity during pregnancy and in the postpartum is safe, has benefits to the health of the mother and baby, and reduces the risks of some complications related to pregnancy. Pregnancy should be encouraged to do at least 150 minutes a week of moderate intensity physical activity. Pregnancy women with contraindications should seek help from qualified professionals. In some cases, physical activity during pregnancy is not recommended. This document will be used as a tool to guide health professionals who work with pregnant women and women in the postpartum period. Also, it will inform and guide women during pregnancy and postpartum about the benefits of physical activity.

Keywords: Physical activity; Pregnancy; Exercise; Population. 


\section{Introdução}

A atividade física desempenha um papel importante na saúde materno-infantil. Uma série de estudos tem demonstrado que a participação em atividades físicas de lazer durante a gestação está associada a melhores resultados de saúde para a mãe e para o bebê $\hat{e}^{1,2}$. Além disso, a atividade física deve ser considerada uma terapia de primeira linha para reduzir o risco de complicações durante a gestação e melhorar a saúde física e mental materna ${ }^{3}$.

Desde as primeiras orientações para exercícios pré-natais que foram desenvolvidas pelo American College of Obstetricians and Gynecologists em 1985', um crescente corpo de evidências tem demonstrado a segurança e os benefícios da atividade física para gestantes ${ }^{1-3,5,6}$. Contudo, apesar dessas recomendações estarem documentadas $^{7}$, popularmente ainda existe uma preocupação sobre o fato de que a prática de atividade física possa prejudicar a saúde da gestante e do bebê. Dessa forma, são necessários esforços de órgãos governamentais para que sejam criadas estratégias de disseminação de informações baseadas em evidências para profissionais de saúde a fim de que a população seja melhor amparada.

A elaboração do Guia de Atividade Física para a População Brasileira, com espaço específico para recomendações direcionadas às gestantes e mulheres no período pós-parto, objetiva preencher um importante hiato existente entre o conhecimento científico e o uso prático das evidências mais confiáveis no Brasil. Dessa forma, o objetivo principal desse documento, direcionado a profissionais de saúde que atuam diariamente com este público, é apresentar as recomendações de atividade física para gestantes e puérperas desenvolvidas para o Guia de Atividade Física para a População Brasileira. O objetivo secundário desde manuscrito é sintetizar o processo metodológico utilizado para construção do Guia de Atividade Física para a População Brasileira voltado para gestantes e puérperas.

\section{Métodos}

O planejamento e a execução das atividades para a construção das recomendações de atividade física para gestantes e mulheres no pós-parto do Guia de Atividade Física para a População Brasileira foi conduzido por um Grupo de Trabalho com sete pesquisadores e dois representantes do Ministério da Saúde. As recomendações foram desenvolvidas utilizando uma abordagem de métodos mistos. Inicialmente foi realizado um le- vantamento das diretrizes internacionais mais recentes ${ }^{7}$, incluindo as recomendações de atividade física do Canadá ${ }^{2}$ Estados Unidos ${ }^{1,8}$ e do Colégio Americano de Ginecologistas e Obstetras ${ }^{3,9}$. Posteriormente, para atualização das evidências consideradas pelas diretrizes, foi realizada uma busca sistemática por ensaios clínicos randomizados, estudos de coorte e revisões sistemáticas e metanálises publicados entre janeiro de 2018 e junho de 2020.

A partir da sumarização das evidências encontradas, foram identificados desfechos-chave relacionados a saúde da mulher durante a gestação, ao desenvolvimento e saúde da criança, ao parto, além de desfechos relacionados ao pós-parto. Para cada um dos desfechos identificados o efeito da atividade física foi classificado como: i) positivo; ii) negativo; ou iii) neutro. A qualidade da evidência sobre os efeitos da atividade física em cada um dos desfechos foi avaliada por meio do Grading of Recommendations Assessment, Development and Evaluation (GRADE) ${ }^{10}$. Esse sistema classifica a qualidade da evidência em quatro níveis: alta, moderada, baixa ou muito baixa. O nível e qualidade da evidência sobre os efeitos da atividade física para cada um dos desfechos identificados foi avaliado de forma independente por dois integrantes do Grupo de Trabalho. As inconsistências entre avaliadores foram discutidas e resolvidas de forma consensual pelos integrantes do Grupo de Trabalho.

Em paralelo ao processo de identificação e avaliação das evidências sobre os efeitos da atividade física durante a gestação, foi realizado um processo de escuta sobre atividade física na gestação e no pós-parto. Esta etapa teve como objetivo identificar dificuldades, facilitadores, estratégias, 'mitos' e opiniões de profissionais de saúde e mulheres sobre conteúdos importantes a serem incorporados no Guia de Atividade Física para a População Brasileira.

Este processo de escuta incluiu um formulário eletrônico e rodas de conversa com gestantes e mulheres no período pós-parto. $\mathrm{O}$ formulário eletrônico, contendo questões fechadas e abertas, foi utilizado para coletar informações de conhecimento e opiniões sobre recomendações e prática de atividade física das partes interessadas (gestantes, mulheres no pós-parto, pesquisadores brasileiros e profissionais, incluindo profissionais de saúde vinculados ao Programa Academia da Saúde). O convite para o preenchimento do formulário foi disseminado via e-mail, redes sociais (Instagram e Facebook) e aplicativo de mensagens gratuito (Whats $A$ - 
$p p)$ para as pessoas da população-alvo, mediante indicações. As rodas de conversa com gestantes e mulheres no pós-parto foram realizadas de maneira virtual e de diferentes regiões do Brasil. Essas mulheres foram indicadas como possíveis candidatas para participar das rodas por integrantes do Grupo de Trabalho que trabalharam na construção do Guia de Atividade Física para a População Brasileira.

Todos os participantes acessaram uma carta convite, em seguida confirmaram o aceite em participar do processo de escuta por meio de uma declaração de confidencialidade e conflito de interesses, preenchendo: nome completo, CPF, e-mail, cidade e estado. Detalhes metodológicos relacionados ao processo de revisão, avaliação de evidências e escuta com a população estarão disponíveis online no endereço eletrônico do Ministério da Saúde (www.saude.gov.br).

Por fim, foi realizada uma consulta pública da versão final do documento, em agosto de 2020. O texto e um formulário online onde a população poderia registrar sugestões para a redação do Guia foram hospedados no website do Ministério da Saúde. Foram recebidas 25 sugestões válidas. Após o período de consulta pública, as sugestões recebidas foram analisadas pelo Grupo de Trabalho e, 13 delas foram consideradas com relevância técnica e/ou social e incorporadas ao texto; enquanto 12 não foram incorporadas e por isso, justificadas.

\section{Resultados}

As evidências sumarizadas suportam que a prática de atividade física durante a gestação e no período pós- parto é segura, traz benefícios à saúde da mãe e do bebê, e reduz os riscos de algumas complicações relacionadas à gestação. Um resumo com as principais recomendações sobre a prática de atividade física $\mathrm{du}-$ rante a gestação e no período pós-parto é apresentado no Quadro 1. Detalhamento metodológico e a lista completa com estudos identificados durante o processo de revisão estão disponíveis online no relatório desenvolvido pelo Grupo de Trabalho.

\section{Atividade física durante a gestação e saúde da mulher}

Gestantes saudáveis, sem contraindicações, podem e devem praticar atividade física regular para alcançar benefícios físicos e mentais, assim como evitar ou minimizar complicações de saúde $e^{1,3,5}$.

Os efeitos da atividade física durante a gestação e a qualidade das evidências disponíveis para desfechos
Quadro 1 - Recomendações de atividade física durante a gestação e período pós-parto para o Guia de Atividade Física para a População Brasileira.

\begin{tabular}{|l|}
\hline \multicolumn{1}{|c|}{ Recomendações gerais } \\
\hline - Gestantes sem contraindicação podem acumular entre 150 e 300 \\
minutos por semana de atividade física com intensidade moderada. \\
Gestantes sem contraindicação e que realizavam atividade física antes da \\
gestação também podem praticar entre 75 e 150 minutos por semana de \\
atividade física com intensidade vigorosa. \\
- Mulheres que já eram fisicamente ativas antes da gestação podem \\
manter a rotina de atividade física. Mulheres que não eram ativas antes \\
da gestação podem iniciar a prática de atividade física de forma gradual \\
em qualquer momento da gestação. \\
- Mulheres que não eram fisicamente ativas antes da gestação são \\
aconselhadas a realizar atividades de intensidade leve a moderada. \\
Mulheres que eram ativas antes da gestação podem continuar com a \\
rotina de atividades físicas e realizar atividades de intensidade moderada \\
a vigorosa durante a gestação. \\
- O trabalho multidisciplinar é fundamental para o sucesso de uma \\
gestação. Todos os profissionais de saúde que prestam assistência durante \\
a gestação devem estar familiarizados com as contraindicações, sinais e \\
sintomas que sugerem que a rotina de atividade física seja modificada ou \\
interrompida. \\
- O apoio da família e amigos é fundamental para que mulheres possam \\
praticar atividade física durante a gestação e o período pós-parto. \\
- Exercícios para o assoalho pélvico são recomendados e podem ser \\
iniciados logo após o parto.
\end{tabular}

relacionados à saúde da mulher são apresentados no Quadro 2. De uma forma geral, há evidências de qualidade alta mostrando que a prática de atividade física durante a gestação diminui o risco de ganho de peso excessivo e o desenvolvimento de hipertensão gestacional ${ }^{11}$. Existem evidências moderadas sugerindo que a prática de atividade física durante a gestação ajuda a manter a aptidão cardiorrespiratória ${ }^{12}$, diminui o risco de diabetes gestacional e pré-eclâmpsia ${ }^{9}$. Gestantes que fazem exercícios para os músculos do assoalho pélvico durante e após a gestação com auxílio de profissionais de saúde têm menos risco de desenvolver incontinência urinária ${ }^{13,14}$. Entretanto, não existem evidências conclusivas de que programas de exercício que não incluem exercícios específicos do assoalho pélvico tenham efeito na prevenção ou tratamento do quadro de incontinência urinária ${ }^{13}$. A prática regular de atividade física durante a gestação não reduz o risco de dor lombar e pélvica, mas pode ajudar a reduzir sintomas ${ }^{15,16}$. Entretanto, a qualidade desta evidência é muito baixa, sendo originada de estudos experimentais com baixa qualidade ou estudos observacionais suscetíveis a vieses ${ }^{16}$.

Apesar de estarem disponíveis em menor quantidade, evidências de estudos de qualidade moderada, demonstram que a atividade física reduz a gravidade dos sintomas depressivos durante a gestação, diminuindo em até $67 \%$ o risco de depressão nesse período ${ }^{8,17}$. Por 
outro lado, as evidências sugerem que a atividade física durante a gestação não altera o risco de desenvolver ansiedade ou sintomas de ansiedade ${ }^{17}$.

Para atingir os efeitos positivos da atividade física na saúde da mulher, as gestantes devem ser incentivadas a realizar pelo menos 150 minutos por semana de atividade física de intensidade moderada como, por exemplo, caminhada rápida, aulas de hidroginástica, pedalar em bicicleta estacionária e exercícios de força. Parece existir uma relação de dose-resposta para alguns desfechos de saúde, sendo que os volumes de atividade física acima do mínimo recomendado podem causar maiores reduções no risco de desenvolver diabetes gestacional, hipertensão gestacional e pré-eclâmpsia ${ }^{11}$.
Atividade física durante a gestação, parto, desenvolvimento fetal e saúde do bebê

As evidências disponíveis mostram que a prática de atividade física durante a gestação é segura e não apresenta riscos para o desenvolvimento fetal e saúde da criança (Quadro 3). Evidências de qualidade baixa apontam que a prática de atividade física durante a gestação não aumenta o risco de abortos, anomalias congênitas, mortalidade prematura ${ }^{18}$, ruptura da bolsa amniótica antes do início do trabalho de parto, cesariana, indução do trabalho de parto, tempo de parto, laceração do períneo, fadiga, lesão, trauma musculoesquelético e danos maternos, mesmo quando praticada no primeiro trimestre $^{19}$. Também há evidências de qualidade baixa

Quadro 2 - Resumo de evidências do efeito da atividade física sobre a saúde da mulher.

\begin{tabular}{|c|c|c|c|}
\hline Desfecho & $\begin{array}{c}\text { Efeito da } \\
\text { atividade física }\end{array}$ & $\begin{array}{l}\text { Nível de } \\
\text { evidência }\end{array}$ & Observações \\
\hline $\begin{array}{l}\text { Aptidão cardiorrespiratória: } \\
\text { Participação regular em atividade física } \\
\text { durante a gestação ajuda a manter ou } \\
\text { aumentar a aptidão cardiorrespiratória }\end{array}$ & + & Moderado & $\begin{array}{l}\text { A prática de exercício aeróbico regular em mulheres que amamentam } \\
\text { melhora a aptidão cardiorrespiratória materna sem afetar a produção e a } \\
\text { composição do leite }\end{array}$ \\
\hline $\begin{array}{l}\text { Ganho de peso excessivo: } \\
\text { Participação regular em atividade física } \\
\text { durante a gestação ajuda a manter ou } \\
\text { reduz o ganho de peso excessivo }\end{array}$ & + & Alto & $\begin{array}{l}\text { A prática de atividade física de moderada intensidade, realizada entre } \\
\text { 150-300 minutos por semana, reduz o risco do ganho de peso em } \\
\text { excesso durante a gestação }\end{array}$ \\
\hline $\begin{array}{l}\text { Diabetes gestacional: } \\
\text { Prática regular de atividade física durante } \\
\text { a gestação reduz o risco de diabetes } \\
\text { gestacional }\end{array}$ & + & Moderado & $\begin{array}{l}\text { Para alcançar alguma redução no risco de desenvolver diabetes } \\
\text { gestacional, as mulheres devem realizar atividade física pelo menos } 3 \\
\text { vezes por semana, em um mínimo de } 25 \text { minutos por sessão, mas maiores } \\
\text { volumes semanais podem resultar em maiores benefícios. As atividades } \\
\text { podem ser variadas, como caminhada rápida, hidroginástica, ciclismo } \\
\text { estacionário ou treinamento de força }\end{array}$ \\
\hline $\begin{array}{l}\text { Hipertensão gestacional: } \\
\text { Prática regular de atividade física durante } \\
\text { a gestação reduz o risco de hipertensão } \\
\text { gestacional }\end{array}$ & + & Alto & $\begin{array}{l}\text { Para alcançar alguma redução no risco de desenvolver hipertensão } \\
\text { gestacional, as mulheres devem realizar atividade física pelo menos } 3 \\
\text { vezes por semana, em um mínimo de } 25 \text { minutos por sessão, mas maiores } \\
\text { volumes semanais podem resultar em maiores benefícios. As atividades } \\
\text { podem ser variadas, como caminhada rápida, hidroginástica, ciclismo } \\
\text { estacionário ou treinamento de força }\end{array}$ \\
\hline $\begin{array}{l}\text { Pré-eclâmpsia: } \\
\text { Prática regular de atividade física durante a } \\
\text { gestação reduz o risco de pré-eclâmpsia }\end{array}$ & + & Moderado & $\begin{array}{l}\text { Para alcançar alguma redução no risco de desenvolver pré-eclâmpsia, } \\
\text { as gestantes devem realizar atividade física pelo menos } 3 \text { vezes por } \\
\text { semana, em um mínimo de } 25 \text { minutos por sessão, mas maiores volumes } \\
\text { semanais podem resultar em maiores benefícios. As atividades podem ser } \\
\text { variadas, como caminhada rápida, hidroginástica, ciclismo estacionário } \\
\text { ou treinamento de força }\end{array}$ \\
\hline $\begin{array}{l}\text { Dor lombar: } \\
\text { Prática regular de atividade física durante } \\
\text { a gestação não reduz o risco de dor lombar } \\
\text { e pélvica, mas pode ajudar a reduzir } \\
\text { sintomas }\end{array}$ & + & Muito baixo & $\begin{array}{l}\text { Exercícios específicos para estabilização e fortalecimento dos músculos } \\
\text { glúteos, extensores e abdutores do quadril, bem como a educação das } \\
\text { gestantes sobre a importância de exercícios e alongamentos seguros, } \\
\text { podem ajudar a atenuar a gravidade dos sintomas de dor lombar e } \\
\text { pélvica. O risco de dor pode ser maior em gestantes obesas }\end{array}$ \\
\hline $\begin{array}{l}\text { Incontinência urinária: } \\
\text { Prática regular de exercícios para o } \\
\text { assoalho pélvico reduz o risco e sintomas } \\
\text { de incontinência urinária durante a } \\
\text { gestação, ou no pós-parto }\end{array}$ & + & Moderada & $\begin{array}{l}\text { Gestantes devem ser aconselhadas a fazer exercícios para os músculos do } \\
\text { assoalho pélvico durante e após a gestação com auxílio de profissionais } \\
\text { de saúde }\end{array}$ \\
\hline $\begin{array}{l}\text { Depressão e ansiedade: } \\
\text { Participação regular em atividade física } \\
\text { durante a gestação reduz o risco e os } \\
\text { sintomas de depressão }\end{array}$ & $\mathrm{N}$ & Moderado & $\begin{array}{l}\text { A prática de atividade física durante a gestação parece não alterar o risco } \\
\text { da mulher desenvolver ansiedade ou sintomas de ansiedade }\end{array}$ \\
\hline
\end{tabular}

+ = efeito positivo; - = efeito negativo; $\mathrm{N}$ = sem efeito. 
sugerindo que não praticar atividade física desde o primeiro trimestre aumenta os riscos de complicações relacionadas à gestação. Evidências de qualidade moderada mostram que a prática de atividade física durante a gestação não afeta o crescimento fetal. Além do mais, evidências de qualidade moderada indicam que a prática de atividade física durante a gestação está associada a redução de risco de nascimentos prematuros e de bebês acima do peso para a idade gestacional ${ }^{2}$. Existe recomendação forte e qualidade da evidência moderada, que atividade física na gestação está associada com redução do risco de macrossomia, e de outros resultados adversos incluindo parto prematuro e baixo peso ao nascer ${ }^{20}$. Apesar de alguns estudos sugerirem que a atividade física durante gestação está associada a menor peso ao nascer nas crianças, as diferenças no peso ao nascer encontradas são mínimas e não impactam em baixo peso ao nascer de acordo com os parâmetros esperados. Evidências de muito baixa qualidade indicam que a prática de atividade física durante a gestação não impacta no escore médio do APGAR $^{20,21}$.

\section{Atividade física e pós-parto}

O período pós-parto é um momento oportuno para que um estilo de vida ativo e saudável seja adotado ${ }^{3}$. Contudo, a retomada das práticas anteriores ou adesão a outras, relacionadas ao estilo de vida, pode ser um desafio ${ }^{3}$. $\mathrm{Na}$ ausência de complicações médicas ou cirúrgicas relacionadas ao parto, a prática de atividade física pode ser retomada gradualmente após a gestação. Algumas mulheres são capazes de retomar as atividades físicas alguns dias após o parto.

A prática de atividade física no pós-parto tem efeitos positivos na saúde da mulher, além de não apresentar efeitos negativos em relação a amamentação (Quadro 4). Recomenda-se que, neste período, pratiquem pelo menos 150 minutos por semana de atividade física de intensidade moderada. Mulheres que habitualmente praticam atividade vigorosa ou, que eram fisicamente ativas antes da gestação podem continuar essas atividades durante o período pré-natal e pós-parto ${ }^{3,8}$. É importante destacar que a prática regular de atividade física em mulheres lactantes melhora a aptidão cardiovascular materna sem afetar a produção e composição do leite ${ }^{22}$.

Evidências sugerem que as mulheres ativas são menos propensas a apresentarem depressão no pós-parto quando comparadas com as menos ativas ${ }^{8}$. Além disso, evidências de alta qualidade sugerem que a prática de atividade física auxilia a mulher a retornar ao peso anterior à gestação. Intervenções durante a gestação podem auxiliar na menor retenção de peso um ano após o parto. Todavia, é preciso fazer algumas ponderações em relação aos resultados apresentados quanto à redução de peso no pós-parto, sobretudo considerando dados publicados anteriormente ${ }^{1,8}$. Os documentos ressaltam que as evidências são insuficientes para determinar qual dose ideal de atividade física para perda de peso durante o pós-parto e também para determinar se existe uma

Quadro 3- Resumo de evidências do efeito da atividade física sobre a saúde do bebê.

\begin{tabular}{|c|c|c|c|}
\hline Desfecho & Efeito & Nível de evidência & Observações \\
\hline $\begin{array}{l}\text { Prematuridade: } \\
\text { A prática de atividade física durante a } \\
\text { gestação reduz o risco de prematuridade }\end{array}$ & + & Moderado & $\begin{array}{l}\text { Atividade física durante a gestação reduz a incidência de prematuridade, até } \\
\text { mesmo em mulheres obesas, quando praticada de } 3 \text { a } 7 \text { vezes por semana, com } \\
\text { duração de } 30 \text { a } 60 \text { minutos }\end{array}$ \\
\hline $\begin{array}{l}\text { Crescimento fetal: } \\
\text { A participação em atividade física afeta o } \\
\text { crescimento fetal }\end{array}$ & $\mathrm{N}$ & Moderado & $\begin{array}{l}\text { A prática regular de atividade física durante a gestação parece não afetar o } \\
\text { crescimento fetal }\end{array}$ \\
\hline $\begin{array}{l}\text { Peso ao nascer: } \\
\text { A prática de atividade física durante a } \\
\text { gestação reduz o risco de macrossomia }\end{array}$ & + & Moderado & $\begin{array}{l}\text { A atividade física durante a gestação reduz o risco de macrossomia (excesso de } \\
\text { peso ao nascer) }\end{array}$ \\
\hline $\begin{array}{l}\text { Aborto: } \\
\text { A atividade física praticada de forma } \\
\text { regular durante a gestação reduz o risco } \\
\text { de abortos }\end{array}$ & $\mathrm{N}$ & Baixo & $\begin{array}{l}\text { Evidências apontam que a prática de atividade física durante a gestação não } \\
\text { aumenta o risco de abortos. Mesmo quando praticada no primeiro trimestre } \\
\text { da gestação não aumenta a probabilidade de aborto ou anomalias congênitas }\end{array}$ \\
\hline $\begin{array}{l}\text { Mortalidade perinatal: } \\
\text { A prática regular de atividade física } \\
\text { durante a gestação não aumenta o risco } \\
\text { de mortalidade perinatal }\end{array}$ & $\mathrm{N}$ & Baixo & $\begin{array}{l}\text { A prática regular de atividade física durante a gestação não aumenta o risco de } \\
\text { incidência de mortalidade perinatal }\end{array}$ \\
\hline $\begin{array}{l}\text { APGAR: } \\
\text { A prática de atividade física durante a } \\
\text { gestação pode aumentar o APGAR }\end{array}$ & $\mathrm{N}$ & Baixo & $\begin{array}{l}\text { Não existem evidências de que a prática de atividade física durante a gestação } \\
\text { aumenta ou diminui os escores médio do APGAR aos } 5 \text { ou } 7 \text { minutos }\end{array}$ \\
\hline
\end{tabular}

+ = efeito positivo; - = efeito negativo; $\mathrm{N}$ = sem efeito. 
relação dose-resposta entre atividade física e perda de peso durante o pós-parto ${ }^{8}$. Além do mais, a realização de treinamento muscular do assoalho pélvico ${ }^{23}$ pode ser realizado diariamente para reduzir o risco de incontinência urinária após o parto.

\section{Intensidade da atividade física}

As evidências existentes indicam que as gestantes saudáveis podem iniciar ou manter atividade física regular com intensidade moderada (quando previamente inativas) ou intensidade vigorosa (quando previamente ativas) $)^{3,5,23}$. Atividade física de intensidade vigorosa até o terceiro trimestre parece ser segura para a maioria das gestações saudáveis. Entretanto, mais pesquisas sobre os efeitos da atividade física de intensidade vigorosa são necessárias, particularmente estudos que envolvam exercícios com intensidade superior a $90 \%$ da frequência cardíaca máxima no primeiro e segundo trimestre. Pode ser mais seguro sugerir que apenas exercícios de intensidade moderada sejam realizados no terceiro trimestre ${ }^{23}$.

A intensidade da atividade física durante a gestação e pós-parto pode ser monitorada pela percepção de esforço e o teste da fala. Dessa forma, numa escala de 0 a 10, a percepção de cansaço para atividades físicas de intensidade moderada estará entre 5 e 6, tornando uma conversação difícil. Para atividades vigorosas, a percepção estará entre 7 e 8 e irão incluir atividades nas quais a mulher não irá conseguir conversar enquanto se movimenta.

\section{Contraindicações e riscos}

De uma forma geral, as evidências mostram consistentemente que mulheres com gestação saudável e sem contraindicações podem realizar atividades físicas simples, como por exemplo a caminhada em intensidades leves e moderadas. Entretanto, algumas precauções devem ser tomadas, especialmente com atividades que envolvam riscos de queda ou contato físico intenso com pessoas e objetos (por exemplo: futebol, basquete, lutas). Deve-se evitar a realização de atividades físicas em calor excessivo, especialmente com alta umidade (por exemplo: saunas), com intuito de evitar a hipertermia, principalmente no primeiro trimestre ${ }^{5,9}$.

Existem situações que a prática de atividade física é contraindicada durante a gestação ${ }^{24}$. Assim sugere-se que na presença de algum dos fatores listados no Quadro 5, a prática de atividade física durante gestação precisa ser indicada por um profissional de saúde que acompanhe o pré-natal e orientada por um profissional capacitado, incluindo profissionais de educação física e fisioterapeutas que tenham experiência com a orientação de atividades físicas para gestantes. $\mathrm{O}$ acompanhamento profissional tem como objetivo manter a segurança da mãe e do bebê.

\section{Atividade física e adaptações fisiológicas durante a gestação}

Diversas mudanças anatômicas e fisiológicas ocorrem durante a gestação. Tais adaptações apresentam variação com relação a idade gestacional e refletem nas taxas de crescimento do bebê e nas mudanças do ganho de peso gestacional ${ }^{25}$. Todas essas adaptações devem ser consideradas para a prática da atividade física ${ }^{3}$.

Entre inúmeras mudanças na gestação, as mais distintas são o ganho de peso corporal e a mudança

Quadro 4- Resumo de evidências do efeito da atividade física sobre a saúde da mulher no pós-parto.

\begin{tabular}{|c|c|c|c|}
\hline Desfecho & Efeito & Nível de evidência & Observações \\
\hline $\begin{array}{l}\text { Retenção de peso: } \\
\text { Prática regular de atividade física } \\
\text { contribui para a mulher retornar ao } \\
\text { peso de antes de engravidar e, quando } \\
\text { necessário, uma redução adicional de peso }\end{array}$ & + & Alto & $\begin{array}{l}\text { A prática de atividade física de moderada intensidade, realizada por, no } \\
\text { mínimo, } 150 \text { minutos por semana ou a prática de atividade física de vigorosa } \\
\text { intensidade, realizada por, no mínimo, } 75 \text { minutos por semana, auxilia no } \\
\text { manejo de peso após a gestação. }\end{array}$ \\
\hline $\begin{array}{l}\text { Amamentação: } \\
\text { Prática regular de atividade física não } \\
\text { prejudica a lactação }\end{array}$ & $\mathrm{N}$ & Baixo & $\begin{array}{l}\text { A prática de atividade física e lactação são compatíveis. Mulheres no pós-parto } \\
\text { podem praticar atividade física regularmente, pois, parece não influenciar na } \\
\text { amamentação }\end{array}$ \\
\hline $\begin{array}{l}\text { Incontinência urinária: } \\
\text { Prática regular de exercícios para o } \\
\text { assoalho pélvico reduz o risco e sintomas } \\
\text { de incontinência urinária no período } \\
\text { pós-parto }\end{array}$ & + & Moderado & $\begin{array}{l}\text { Mulheres no pós parto são aconselhadas a fazer exercícios para os músculos } \\
\text { do assoalho pélvico. Estes exercícios podem ser realizados de forma isolada ou } \\
\text { combinada com outros exercícios, por exemplo, aeróbicos }\end{array}$ \\
\hline $\begin{array}{l}\text { Depressão e ansiedade: } \\
\text { Prática regular de atividade física } \\
\text { durante o pós-parto não reduz o risco de } \\
\text { depressão ou sintomas de ansiedade }\end{array}$ & $\mathrm{N}$ & Moderado & $\begin{array}{l}\text { A prática de atividade física durante o pós parto parece não alterar o risco de a } \\
\text { mulher desenvolver depressão ou sintomas de ansiedade }\end{array}$ \\
\hline
\end{tabular}

+ = efeito positivo; - = efeito negativo; $\mathrm{N}$ = sem efeito. 
do centro de gravidade, o que resulta em uma lordose progressiva. Essas alterações levam a um aumento da carga articular na coluna vertebral, podendo gerar dores, principalmente lombares ${ }^{3,26}$. Atividades físicas para fortalecer os músculos abdominais e das costas podem reduzir o risco do surgimento de dor lombar ${ }^{26}$.

Algumas posições corporais podem ser incômodas para a gestante por conta das alterações anatômicas. Atividades físicas na posição supina após a $20^{\mathrm{a}}$ semana podem resultar em uma diminuição do retorno venoso devido a uma compressão da aorta e das veias cavas, resultante do útero gravídico, proporcionando hipotensão e mudanças hemodinâmicas que devem ser consideradas para prática dos movimentos corporais ${ }^{3}$. Apesar de estudos relatarem uma bradicardia e alterações no padrão de frequência cardíaca fetal em atividades realizadas na posição supina, nenhum resultado adverso foi verificado, indicando ainda falta de evidências sobre o assunto ${ }^{27}$. Em algumas gestantes pode ocorrer uma separação dos músculos abdominais. Nesses casos, gestantes são aconselhadas a buscar aconselhamento profissional e evitar exercícios de fortalecimento abdominal ${ }^{5}$.

Entre as mudanças cardiovasculares que ocorrem na gestação, observa-se principalmente o aumento do volume sanguíneo, da frequência cardíaca, do volume de ejeção, do débito cardíaco e a redução da resistência vascular sistêmica ${ }^{3,28}$. O bebê em crescimento precisa de oxigênio, substratos suficientes para seu crescimento e desenvolvimento, manutenção térmica e remoção de subprodutos. Durante a gestação o esforço físico cria um efeito natural e a entrega de substratos fetais se mantém. A atividade física é segura, pois durante e após a prática são estabelecidas reservas circulatórias necessárias para a gestante e para o bebê $\hat{e}^{12,25}$.

A prática regular de atividades físicas pode contribuir com a regulação da pressão arterial, redução do risco de desenvolvimento de hipertensão e pré-eclâmpsia $^{11,28}$. Além disso, melhora o crescimento e a vascularização da placenta, promovendo eficiência no transporte de nutrientes para o desenvolvimento do bebê, e pode melhorar a viscosidade do volume tecidual da placenta ${ }^{29}$.

As respostas cardiopulmonares em repouso, em gestantes bem treinadas, da $35^{\mathrm{a}}$ a $38^{\mathrm{a}}$ semanas podem ser semelhantes a não grávidas que se exercitam. Respostas ventilatórias durante o exercício em grávidas e não grávidas podem ser similares, no entanto, em repouso as mulheres grávidas apresentam maior ventilação minuto do que as não grávidas ${ }^{25}$.

Durante a gestação são percebidas adaptações profundas na ventilação pulmonar. A ventilação minuto aumenta em até $50 \%$, primariamente como um resultado do aumento do volume corrente. Ocorre uma redução do volume de reserva pulmonar, o que prejudica a capacidade de realizar atividades físicas anaeróbias e reduz a capacidade de trabalho em atividades aeróbicas. Atividades físicas aeróbicas na gestação podem trazer adaptações respiratórias benéficas e dessa forma devem ser incentivadas quando não há contraindicação ${ }^{3}$.

Quadro 5 - Contraindicações para a prática de atividade física durante a gestação*.

\begin{tabular}{|l|l|}
\hline $\begin{array}{l}\text { Prática de atividade física deve ser acompanhada por um profissional } \\
\text { capacitado }\end{array}$ & Prática de atividade física é contraindicada \\
\hline Doenças respiratórias leves controladas & $\begin{array}{l}\text { Doenças respiratórias graves (ex. doença pulmonar obstrutiva crônica, doença } \\
\text { pulmonar restritiva e fibrose cística) } \\
\text { Doenças cardíacas leves, congêneas graves com intolerância ao exercício (congênitas ou } \\
\text { Diabetes tipo 1, desde que bem controlada } \\
\text { adquiridas) }\end{array}$ \\
$\begin{array}{l}\text { Pré-eclâmpsia leve } \\
\text { gupturação) }\end{array}$ & $\begin{array}{l}\text { Arritmia grave ou descontrolada } \\
\text { Placenta prévia após } 28 \text { semanas de gestação }\end{array}$ \\
$\begin{array}{l}\text { Doença de tireoide não tratada } \\
\text { Transtornos alimentares sintomáticos }\end{array}$ & $\begin{array}{l}\text { Vasa prévia } \\
\text { Restrição no crescimento intrauterino }\end{array}$ \\
$\begin{array}{l}\text { Deficiências nutricionais ou desnutrição } \\
\text { Uso excessivo de cigarros (acima de } 20 \text { cigarros/dia) na presença de } \\
\text { comorbidades (ex. hipertensão, diabetes) }\end{array}$ & Trabalho de parto prematuro ativo \\
\hline
\end{tabular}

* Adaptado com base em recomendações internacionais ${ }^{3,5}$ e revisão de literatura desenvolvida por Meah et $\mathrm{al}^{24}$. 
Barreiras e facilitadores para a prática de atividade física durante e após a gestação Apesar dos conhecidos benefícios da atividade física na saúde da gestante e do bebê, grande parte das gestantes não atingem as recomendações ${ }^{30,31}$. Ao longo dos trimestres de gestação a mulher reduz a quantidade de atividade física praticada e o mesmo ocorre no pós-parto, principalmente no primeiro ano. Inúmeras barreiras percebidas pelas gestantes podem justificar a falta de engajamento em uma vida ativa. As mais comuns estão relacionadas a própria gestante ${ }^{32}$. Os sintomas e limitações, como cansaço, fadiga, náusea, dor física e alterações corporais, diminuem a motivação e se tornam impedimentos para as mulheres serem ativas durante a gestaçã $0^{33}$. Da mesma forma, também é recorrente o medo de prejudicar o bebê ou a si própria ${ }^{33}$. Essas inseguranças sobre a prática de atividade física na gestação estão relacionadas as barreiras interpessoais, como a falta de aconselhamento e informações adequadas, a falta de apoio social e o desconhecimento de orientações sobre como se exercitar durante a gestação $0^{32,33}$. Além disso, a falta de tempo é uma barreira mencionada pelas gestantes em geral, mas mais frequentemente entre mães não primogênitas e em uma posição de baixo nível socioeconômico, devido às demandas $\mathrm{e}$ compromissos familiares ${ }^{32}$. Da mesma forma, barreiras relacionadas às questões ambientais, como clima desfavorável, acesso limitado a instalações adequadas e seguras, bem como a preocupação com os custos e a falta de programas específicos para gestantes, são proeminentes em mulheres com nível socioeconômico baixo ${ }^{33}$. Por fim, no período pós-parto, algumas das principais barreiras são a falta de tempo, a falta de suporte para cuidar do bebê na ausência da mãe e o cansaço ${ }^{34}$.

Ao contrário das barreiras percebidas, facilitadores podem aumentar e fortalecer o engajamento de gestantes e puérperas na prática de atividade física. Mulheres com maior nível de escolaridade, que em muitos casos têm maior acesso aos facilitadores, são comumente mais ativas ${ }^{32}$. Os fatores relacionados às próprias gestantes são fortes facilitadores, como uma boa saúde materna e do bebê, bem-estar físico, desconfortos da gestação amenizados, estresse reduzido e uma boa autoestima e forma física ${ }^{32}$. Ainda, o acesso a informações sobre benefícios e como praticar atividade física, o suporte social e $\mathrm{o}$ acesso a instalações seguras e adequadas facilitam uma gestação mais ativa. De maneira similar, no período pós-parto alguns dos principais facilitadores são o suporte do companheiro(a) para a prática de atividade física, bem como o suporte social da família e amigos, além dos benefícios de bem-estar físico e mental que são proporcionados pela atividade física ${ }^{34}$.

\section{Discussão}

Neste artigo apresentamos um resumo das evidências sobre os efeitos da atividade física durante a gestação e pós-parto, e sintetizamos o processo de trabalho de elaboração das recomendações de atividade física para gestantes e mulheres no pós-parto. As melhores evidências disponíveis mostram que a prática de atividade física durante a gestação e no período pós-parto é segura, traz benefícios à saúde da mãe e do bebê, e reduz os riscos de algumas complicações relacionadas à gestação. Gestantes devem ser incentivadas a realizar pelo menos 150 minutos por semana de atividade física de intensidade moderada. Esta recomendação pode ser alcançada de maneira progressiva e as atividades podem ser distribuídas em diferentes dias da semana, por exemplo, 15 minutos em 5 dias. As atividades podem ser variadas, como caminhada, corrida, pedalada, dança, exercícios de força com ou sem a criança, entre outros. Estas atividades podem ser realizadas em casa, ao ar livre ou em lugar especializado. Gestantes com contraindicações devem procurar auxílio de profissionais qualificados. Em alguns casos, a prática de atividade física durante a gestação não é recomendada.

O Guia de Atividade Física para a População Brasileira direcionado para gestantes e mulheres no período pós-parto foi construído por meio de um processo rigoroso e interativo que considerou as evidências científicas mais recentes, assim como a diversidade de características culturais e sociais encontradas no Brasil. No presente documento, são apresentadas recomendações de atividade física para gestantes e mulheres no pós- parto, as quais devem ser amplamente utilizadas por profissionais e gestores de saúde que trabalham com este grupo populacional.

Além de auxiliar gestantes e mulheres no pós-parto, as recomendações elaboradas podem embasar as ações de gestores e profissionais de saúde para a inclusão de atividade física na atenção primária, na comunidade e na rotina de atendimento. No presente artigo apresentamos de forma resumida recomendações, contraindicações, barreiras e facilitadores para a prática de atividade física durante a gestação e pós-parto. Entretanto, o Guia de Atividade Física para a População Brasileira direcionado para gestantes e mulheres no pós-parto apresenta as diferentes possibilidades e doses de ativi- 
dades físicas que podem ser realizadas, as contraindicações, bem como as barreiras para a prática com sugestões de como superá-las. Ressaltando que, assim como outros determinantes sociais de saúde, a atividade física deve ser incluída no plano de atuação dos gestores e profissionais de saúde como medida de prevenção de doenças, promoção e manutenção da saúde.

Especificamente, o gestor pode contribuir com a disseminação do material para as gestantes e mulheres no pós-parto, profissionais de saúde e entre os demais gestores, que também serão multiplicadores. Além disso, a partir do Guia, o gestor poderá planejar e implementar ações estratégicas pontuais ou de longo prazo com a sua equipe. Já os profissionais de saúde poderão se beneficiar com material por meio de uma maior segurança para indicar atividades físicas, com embasamento teórico e sugestões práticas, além de serem fundamentais para a divulgação do material para a população. Para ambos, gestores e profissionais de saúde, os parâmetros indicados pelo Guia podem servir de suporte para a realização de avaliação e monitoramento dos níveis de atividade física da população, o que impacta em um melhor planejamento em saúde.

Mesmo que nos últimos anos as evidências sobre o tema tenham aumentado, ainda há necessidade de investigar estratégias que aumentem a adesão das gestantes aos programas de atividade física. Essas estratégias, precisam ser investigadas principalmente, em grupos com condições socioeconômicas distintas. São necessários mais estudos para uma melhor compreensão dos riscos e benefícios de atividades realizadas em intensidade mais vigorosa para as gestantes, assim como não há muitas evidências comparando benefícios entre distintas modalidades de exercício físico. Por fim, as evidências sobre o efeito da atividade física em desfechos de dor lombar/pélvica, incontinência urinária, ansiedade e amamentação durante a gestação e no pós-parto, assim como desfechos de desenvolvimento fetal e trabalho de parto, precisam ser fortalecidas.

O presente documento apresenta recomendações sobre a prática de atividade física para gestantes e mulheres no pós-parto, orientando quanto ao tipo, intensidade e volume adequado para se obter benefícios à saúde. Adicionalmente, com base em ampla revisão da literatura científica apresentada sob quais condições clínicas a gestante pode continuar com a prática de atividade física e em quais a prática de atividade física é desaconselhada. Além disso, apresenta informações sobre os motivos para praticar, como adotar um estilo de vida ativo e saudável e orienta profissionais de saúde para possíveis adaptações, caso sejam necessárias. Dessa forma, o Guia de Atividade Física para a População Brasileira com capítulo específico para gestantes e mulheres no pós-parto é um documento completo e atualizado quanto as orientações nele contidas, e direcionado para as particularidades da população brasileira.

\section{Conflito de interesse}

Os autores declaram não haver conflito de interesse.

\section{Financiamento}

Este trabalho integra o Guia Brasileiro de Atividade Física, que foi financiado pelo Ministério da Saúde do Brasil por meio do Termo de Execução Descentralizada n ${ }^{\circ}$ 56/2019 (projeto: 79224219002/2019; processo: 25000.171034/2019-27).

\section{Contribuição dos autores}

Todos os autores participaram da concepção do manuscrito, revisão de literatura, coleta de dados, análise e interpretação dos dados. Todos os autores revisaram e aprovaram o manuscrito.

\section{Referências}

1. Dipietro L, Evenson KR, Bloodgood B, Sprow K, Troiano RP, Piercy KL, et al. Benefits of Physical activity during pregnancy and postpartum: an umbrella review. Med Sci Sports Exerc. 2019;51(6):1292-302.

2. Silva SG, Ricardo LI, Evenson KR, Hallal PC. Leisure-time physical activity in pregnancy and maternal-child health: a systematic review and meta-analysis of randomized controlled trials and cohort studies. Sports Med. 2017;47(2):295-317.

3. Physical Activity and Exercise During Pregnancy and the Postpartum Period: ACOG Committee Opinion, Number 804. Obstet Gynecol. 2020;135(4):e178-88.

4. American College of Obstetrics and Gynecology (ACOG) Technical Bulletin: Exercise During Pregnancy and the Postnatal Period. Washington: ACOG; 1985.

5. Mottola MF, Davenport MH, Ruchat S-M, Davies GA, Poitras VJ, Gray CE, et al. 2019 Canadian guideline for physical activity throughout pregnancy. Br J Sports Med. 2018;52(21):1339-46.

6. Evenson KR, Mottola MF, Artal R. Review of recent physical activity guidelines during pregnancy to facilitate advice by health care providers. Obstet Gynecol Surv. 2019;74(8):481-9.

7. Evenson KR, Barakat R, Brown WJ, Dargent-Molina P, Haruna M, Mikkelsen EM, et al. Guidelines for Physical Activity during Pregnancy: Comparisons From Around the World. Am J Lifestyle Med. 2014;8(2):102-21.

8. United States. Department of Health and Human Services. Physical Activity Guidelines Advisory Committee. 2018 Physical Activity Guidelines Advisory Committee Scientific Report: To the Secretary of Health and Human Services. 2018. 779 p.

9. ACOG Committee Opinion No. 650: Physical Activity and Exercise During Pregnancy and the Postpartum Period. Obstet Gynecol. 2015;126(6):e135-42. 
10. GRADE handbook [Internet]. [cited 2021 Apr 18]. Disponível em: https://gdt.gradepro.org/app/handbook/ handbook.html

11. Davenport MH, Ruchat S-M, Poitras VJ, Jaramillo Garcia A, Gray CE, Barrowman N, et al. Prenatal exercise for the prevention of gestational diabetes mellitus and hypertensive disorders of pregnancy: a systematic review and metaanalysis. Br J Sports Med. 2018;52(21):1367-75.

12. Cai C, Ruchat S-M, Sivak A, Davenport MH. Prenatal exercise and cardiorespiratory health and fitness: a metaanalysis. Med Sci Sports Exerc. 2020 Jul;52(7):1538-48.

13. Davenport MH, Nagpal TS, Mottola MF, Skow RJ, Riske L, Poitras VJ, et al. Prenatal exercise (including but not limited to pelvic floor muscle training) and urinary incontinence during and following pregnancy: a systematic review and meta-analysis. Br J Sports Med. 2018;52(21):1397-404.

14. Woodley SJ, Boyle R, Cody JD, Mørkved S, Hay-Smith EJC. Pelvic floor muscle training for prevention and treatment of urinary and faecal incontinence in antenatal and postnatal women. Cochrane Database Syst Rev. 2017;12:CD007471.

15. Marín-Jiménez N, Acosta-Manzano P, Borges-Cosic M, Baena-García L, Coll-Risco I, Romero-Gallardo L, et al. Association of self-reported physical fitness with pain during pregnancy: The GESTAFIT Project. Scand J Med Sci Sports. 2019;29(7):1022-30.

16. Caputo EL, Ferreira PH, Ferreira ML, Bertoldi AD, Domingues MR, Shirley D, et al. Physical Activity Before or During Pregnancy and Low Back Pain: Data From the 2015 Pelotas (Brazil) Birth Cohort Study. J Phys Act Health. 2019;16(10):886-93.

17. Davenport MH, McCurdy AP, Mottola MF, Skow RJ, Meah VL, Poitras VJ, et al. Impact of prenatal exercise on both prenatal and postnatal anxiety and depressive symptoms: a systematic review and meta-analysis. Br J Sports Med. 2018;52(21):1376-85.

18. Davenport MH, Kathol AJ, Mottola MF, Skow RJ, Meah VL, Poitras VJ, et al. Prenatal exercise is not associated with fetal mortality: a systematic review and meta-analysis. Br J Sports Med. 2019;53(2):108-15.

19. Davenport MH, Ruchat S-M, Sobierajski F, Poitras VJ, Gray $\mathrm{CE}$, Yoo $\mathrm{C}$, et al. Impact of prenatal exercise on maternal harms, labour and delivery outcomes: a systematic review and meta-analysis. Br J Sports Med. 2019;53(2):99-107.

20. Davenport MH, Meah VL, Ruchat S-M, Davies GA, Skow RJ, Barrowman $\mathrm{N}$, et al. Impact of prenatal exercise on neonatal and childhood outcomes: a systematic review and meta-analysis. Br J Sports Med. 2018;52(21):1386-96.

21. Adesegun D, Cai C, Sivak A, Chari R, Davenport MH. Prenatal Exercise and Pre-gestational Diseases: A systematic review and meta-analysis. J Obstet Gynaecol Can. 2019;41(8):1134-43.e17.
22. Cary GB, Quinn TJ. Exercise and lactation: are they compatible? Can J Appl Physiol. 2001;26(1):55-75.

23. Beetham KS, Giles C, Noetel M, Clifton V, Jones JC, Naughton G. The effects of vigorous intensity exercise in the third trimester of pregnancy: a systematic review and metaanalysis. BMC Pregnancy Childbirth. 2019;19(1):281.

24. Meah VL, Davies GA, Davenport MH. Why can't I exercise during pregnancy? Time to revisit medical 'absolute' and 'relative' contraindications: systematic review of evidence of harm and a call to action. Br J Sports Med. 2020;54(23):1395-1404.

25. newton er, may 1 . adaptation of maternal-fetal physiology to exercise in pregnancy: the basis of guidelines for physical activity in pregnancy. Clin Med Insights Womens Health. 2017;10:1179562X17693224.

26. Wang S-M, Dezinno P, Maranets I, Berman MR, CaldwellAndrews AA, Kain ZN. Low back pain during pregnancy: prevalence, risk factors, and outcomes. Obstet Gynecol. 2004;104(1):65-70.

27. Mottola MF, Nagpal TS, Bgeginski R, Davenport MH, Poitras VJ, Gray CE, et al. Is supine exercise associated with adverse maternal and fetal outcomes? A systematic review. $\mathrm{Br}$ J Sports Med. 2019;53(2):82-9.

28. Nagpal TS, Mottola MF. Physical activity throughout pregnancy is key to preventing chronic disease. Reproduction. 2020;160(5):R111-8.

29. Weissgerber TL, Davies GAL, Roberts JM. Modification of angiogenic factors by regular and acute exercise during pregnancy. J Appl Physiol. 2010;108(5):1217-23.

30. Müller WA, Mielke GI, da Silva ICM, Silveira MF, Domingues MR. Physical Activity During Pregnancy and Preterm Birth: Findings From the 2015 Pelotas (Brazil) Birth Cohort Study. J Phys Act Health. 2020;1-10.

31. Mielke GI, Crochemore-Silva I, Domingues MR, Silveira MF, Bertoldi AD, Brown WJ. Physical Activity and Sitting Time From 16 to 24 Weeks of Pregnancy to 12, 24, and 48 Months Postpartum: Findings From the 2015 Pelotas (Brazil) Birth Cohort Study. J Phys Act Health. 2021;1-7.

32. Harrison AL, Taylor NF, Shields N, Frawley HC. Attitudes, barriers and enablers to physical activity in pregnant women: a systematic review. J Physiother. 2018;64(1):24-32.

33. Coll CVN, Domingues MR, Gonçalves H, Bertoldi AD. Perceived barriers to leisure-time physical activity during pregnancy: A literature review of quantitative and qualitative evidence. J Sci Med Sport. 2017;20(1):17-25..

34. Burton C, Doyle E, Humber K, Rouxel C, Worner S, Colman $\mathrm{R}$, et al. The biopsychosocial barriers and enablers to being physically active following childbirth: a systematic literature review. Phys Ther Rev. 2019;24(3-4):143-55.

Recebido: 30/01/2021

Aprovado: 10/05/2021

Como citar este artigo:

Mielke GI, Tomicki C, Botton CE, Cavalcante FVSA, Borges GF, Galliano LM, Sandreschi PF, Pinto SS, Bezerra TA, Hallal PC, Autran R. Atividade física para gestantes e mulheres no pós-parto: Guia de Atividade Física para a População Brasileira. Rev Bras Ativ Fís Saúde. 2021;26:e0217. DOI: 10.12820/rbafs.26e0217 